\title{
DETECTION OF DISTRIBUTED OSCILLATIONS AND ROOT-CAUSE DIAGNOSIS
}

\author{
N.F. Thornhill*, S.L. Shah ${ }^{+}$and B. Huang ${ }^{+}$ \\ *Centre for Process Systems Engineering, Department of Electronic and Electrical \\ Engineering, University College London, Torrington Place, London WC1E 7JE, UK \\ Corresponding author. E-mail: n.thornhill@ee.ucl.ac.uk \\ ${ }^{+}$Department of Chemical and Materials Engineering, University of Alberta, \\ Edmonton, Canada T6G 2G6
}

\section{Abstract}

This article addresses the detection and diagnosis of oscillations in process measurements. Principal components analysis (PCA) can be applied to the power spectra for detection of groups of oscillatory process measurements. In cases where a well defined periodic oscillation is detected then diagnosis of the root cause is possible using a signature that grows stronger closer to the source. Two candidates for the signature are the distortion factor and a non-linearity statistic based on surrogate data. Their performance is compared using experimental data and an industrial data set.

Keywords

Chemical industry; diagnosis; distortion factor; dynamic system; multivariate analysis; non-linearity; plant-wide disturbance; power spectrum; principal components; surrogate data; time series.

\section{Introduction}

It is important to detect and diagnose the causes of oscillations in process operation because a plant running close to a product quality limit is more profitable than a plant that has to back away because of variations in the product (Martin et.al. 1991). Hägglund (1995) described a method for the on-line detection of oscillations within a control loop and other authors have also considered the problem (Rengaswamy and Venkatasubramanian, 1995; Thornhill and Hägglund, 1997). When a disturbance such as oscillation has been detected then it is necessary to find all the measurements or control loops having the same disturbance because the root cause will be among that group. Harris et.al. (1996) reported plant-wide control loop assessment in which spectral analysis was useful. This paper addresses the detection of distributed oscillatory disturbances in cases where the oscillation appears in several measurements, also through analysis of the spectra.

A key issue is to determine the root cause of a plant-wide oscillation (Qin, 1998). It may not be easy to determine cause and effect particularly when physical influences propagate in the opposite direction to process flows, for instance due to recycle streams or when disturbances to the outflow stream of a tank cause deviations in the level of the tank. The core idea in the approach is detection of nonlinearity in the time series of measurements from the process. This paper focuses on the case when the root cause was a non-linear element in a control loop. In a study where the root cause was known, the non-linearity in the time series was found to weaken further away from the source. The reasons for this finding are discussed and an example where the root cause was not known a-priori is then diagnosed.

Once a candidate loop has been identified as the root cause then further analysis or manual testing may be applied to determine the nature of the problem. Thornhill and Hägglund (1997) and Horch (1999) have given procedures for on-line diagnosis of valve and other faults, while McMillan (1995) and Sharif and Grosvenor (1999) reported methods for physical testing of control valves. The benefit of root cause diagnosis is that the maintenance effort required for testing and diagnosis will be directed towards the equipment or control loop that needs it. 


\section{Methods}

\section{Detection of unit-wide disturbances}

In a small study the task of finding all measurements influenced by a plant-wide disturbance may be done by visual inspection. For an automated approach the spectra of the measurements may be compared to one another using principal components analysis (Thornhill et.al, 2000). The data matrix $\boldsymbol{X}$ is:

$$
\begin{gathered}
N \text { frequency channels } \rightarrow \\
\boldsymbol{X}=\left(\begin{array}{ccc}
P_{1}\left(f_{1}\right) & \ldots & P_{1}\left(f_{N}\right) \\
. . & . . & . . \\
P_{m}\left(f_{1}\right) & \ldots & P_{m}\left(f_{N}\right)
\end{array}\right) \begin{array}{c}
\text { m process } \\
\text { variables } \\
\downarrow
\end{array}
\end{gathered}
$$

where $P_{i}\left(f_{j}\right)$ is the power in the $j$ 'th frequency channel of the $i$ 'th measurement. A PCA decomposition reconstructs the $\boldsymbol{X}$ matrix as a sum over $m$ orthonormal basis functions $\boldsymbol{w}_{1}^{\prime}$ to $\boldsymbol{w}_{m}^{\prime}$ which are spectrum-like functions having $N$ frequency channels arranged as a row vector.

$$
\boldsymbol{X}=\left(\begin{array}{c}
t_{1,1} \\
\ldots \\
t_{m, 1}
\end{array}\right) \boldsymbol{w}_{1}^{\prime}+\left(\begin{array}{c}
t_{1,2} \\
\ldots \\
t_{m, 2}
\end{array}\right) \boldsymbol{w}_{2}^{\prime}+\ldots+\left(\begin{array}{c}
t_{1, m} \\
\ldots \\
t_{m, m}
\end{array}\right) \boldsymbol{w}_{m}^{\prime}
$$

The $\boldsymbol{w}_{i}^{\prime}$ - vectors are the normalised eigenvectors of the $m$ by $m$ matrix $\boldsymbol{X}^{\prime} \boldsymbol{X}$. They are ordered according to the size of the eigenvalues of $\boldsymbol{X}^{\prime} \boldsymbol{X}$. The ratio between the eigenvalue and the sum of all the eigenvalues gives a measure of the total spectral variation captured by that eigenvector.

A description of the majority of the variation in $\boldsymbol{X}$ can often be achieved by truncating the PCA description. The following is a three-term PCA model in which the variation of $\boldsymbol{X}$ that is not captured by the first three principal components appears in an error matrix $E$ :

$$
\boldsymbol{X}=\left(\begin{array}{c}
t_{1,1} \\
\ldots \\
t_{m, 1}
\end{array}\right) \boldsymbol{w}_{1}^{\prime}+\left(\begin{array}{c}
t_{1,2} \\
\ldots \\
t_{m, 2}
\end{array}\right) \boldsymbol{w}_{2}^{\prime}+\left(\begin{array}{c}
t_{1,3} \\
\ldots \\
t_{m, 3}
\end{array}\right) \boldsymbol{w}_{3}^{\prime}+\boldsymbol{E}
$$

The above expression shows that the spectra in the $\boldsymbol{X}$ matrix may be approximately reconstructed by a weighted summation of the orthonormal $\boldsymbol{w}^{\prime}-$ vectors.

The issue of the correct number of terms is discussed by Chatfield and Collins (1980), Valle et.al., (1999) and elsewhere. The industrial case study needed three terms, the decision to truncate being made when the eigenvalue associated with the next principal component represented less than $5 \%$ of the sum of all the eigenvalues.
Each spectrum in $\boldsymbol{X}$ may be represented graphically. For instance, when three $\boldsymbol{w}^{\prime}$-vectors are in use the $i$ 'th spectrum maps to a point having the co-ordinates $t_{i, 1}, t_{i, 2}$ and $t_{i, 3}$ in a three-dimensional space known as the scores plot. Similar spectra have similar $t$-coordinates. Therefore such groups form clusters.

\section{Detection of non-linearity}

A common cause of oscillation is the presence of nonlinearity such as a valve dead-band or a faulty instrument which sets up a limit cycle in a control loop (Cook, 1986; Åström, 1991). The oscillation caused by the faulty valve is liable to act as a disturbance elsewhere in the unit. Therefore it is useful to characterise non-linearity in the time trends of the process measurements.

A linear time series (see e.g. Ljung, 1999) has a dynamic model such as the Box Jenkins model with constant coefficients driven by Gaussian white noise. By contrast, the non-linear time series of interest in this work have a non-linear feedback function:

$$
\begin{aligned}
& x(n)=\phi(x(n-1), x(n-2), \ldots ., u(n-1))+w(n) \\
& y(n)=h(x(n))+v(n) \\
& u(n)=g(y(n))
\end{aligned}
$$

where $\phi(x(n-1), x(n-2), \ldots, u(n-1))$ represents linear dynamics, $h(x(n))$ is a measurement function that may be linear or non-linear, $g(x(n))$ is a non-linear feedback function and $w(n)$ and $v(n)$ are process and measurement noises. An example of a non-linear feedback function is the on-off control of a directly-injected steam heated tank in which the steam valve switches on when the temperature drops to a low limit and switches off again when the temperature reaches a high limit. The non-linear characteristic $g$ in that case is a relay with deadband. The temperature is not steady in such a system and it cycles in periodic pattern. Valves with stiction or deadbands and instrumentation faults lead to similar behaviour and are described by the same model structure. An example of a non-linear measurement function would be quantization in the analogue to digital converter in the instrumentation electronics (Goodwin and Welsh, 1999).

\section{Diagnosis using distortion factor analysis}

Non-linearity may be inferred by the presence of a limit cycle. Limit cycles, though periodic, are generally nonsinusoidal and therefore have harmonics at multiples of the fundamental frequency. If plant-wide oscillation is due to limit cycling a candidate for the root cause is the time series with the maximum non-linearity. The reason is that the dynamic behavior of physical processes gives low-pass filtering and therefore removes non-linearity from the time series. As a result, one would expect the harmonics of a 
limit cycle to become smaller further from the root cause and the time trends to become more sinusoidal.

This work used a distortion factor calculation to aid diagnosis of oscillations caused by limit cycles. The distortion factor, $D$, is:

$$
D=\sqrt{\frac{P_{\text {tot }}-P_{\text {fund }}}{P_{\text {tot }}}}
$$

where $P_{t o t}$ is the total power in the fundamental and harmonics and $P_{\text {fund }}$ is the power in frequency channels $k$ to $k+\ell$ occupied by the fundamental harmonic:

Calculation of $D$ requires an inspection of the spectrum to determine a suitable range $k$ to $k+\ell$. In this work the spectra were assessed by hand. The task is not onerous in cases where a cluster of measurements having a common oscillation has been detected because the values of $k$ and $k+\ell$ need be determined only once and can then be used for all the spectra in that cluster. $D$ cannot be determined in cases with no well-defined oscillation and no spectral peak. The measurement having the highest distortion factor has more power in the harmonics and is thus a candidate for the root cause.

\section{Diagnosis using non-linear time series analysis}

The question is whether or not a time series could plausibly be the output of a linear system driven by Gaussian white noise (Theiler et. al., 1992; Kantz and Schreiber, 1997; Heger et.al., 1999; Schreiber and Schmitz, 2000). Many test statistics have been suggested in the literature (Kantz and Schreiber, 1997). The one used here was the r.m.s. value of the error from zero-order nonlinear prediction using matching of nearest neighbors in an $m$-dimensional phase space (for instance, a plot of $x(n)$ versus $x(n-d)$ for some delay $d$ would be a two dimensional phase space). Codes for the calculation were

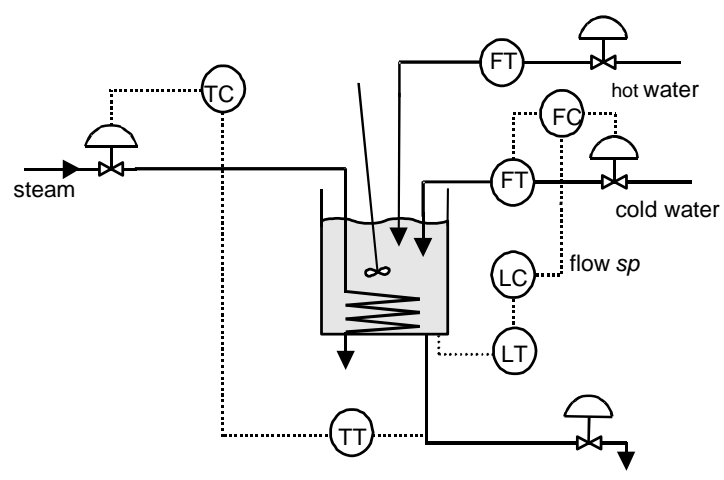

public domain Matlab software by Kaplan (Kaplan, 2000). Numerous random surrogate data sets were derived from the test time series. The surrogate data sets are consistent with the null hypothesis of a linear system driven by Gaussian white noise because they have the same power spectrum and autocorrelation function as the test time series, but differ in the higher order structure. For instance, a time series with a power law non-linearity has a relationship between the phases of the sum and difference frequencies which would not be present in the surrogates.

The prediction errors for the surrogates define a reference probability distribution under the null hypothesis and the prediction error for the time series is compared to that distribution. It is a one-sided hypothesis test because nonlinear effects of the types discussed earlier make the time series more predictable than the surrogates. If the prediction error is an outlier (defined here as lying more than $2 \sigma$ below the mean) then the time series is not consistent with the null hypothesis of a linear process driven by Gaussian white noise. The statistic $N$ was the deviation below the mean in units of $2 \sigma$. Any time series with $N>1$ was classed as non-linear with the probability of a mistake in that diagnosis being less than about $2.5 \%$ if the reference distribution is Gaussian. Larger values of $N$ were interpreted as indicating the time series had more non-linearity, those with $N<1$ were taken to be linear.

\section{Case studies}

\section{A known root cause}

An experimental study at the University of Alberta made a non-linear off-on disturbance to a hot water flow valve in a stirred tank pilot plant. The disturbance is like that from a relay. Figure 1 shows the schematic and the experimental time trends. The scope of the study was small enough that the measurements could be inspected visually. It is known that this plant is well modeled by linear dynamics except that the cold water valve has a mild non-linear relationship
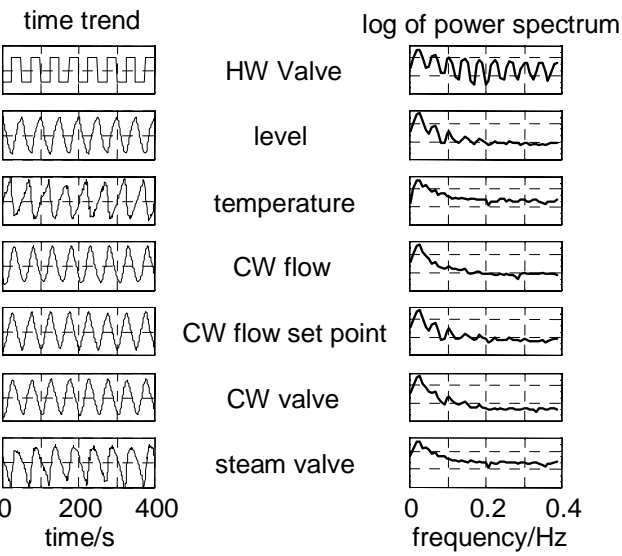

Figure 1. Process schematic and results from experiments 
in its normal operating region (figure 2). Figure 2 shows the full range of the flow meter to be $4-24 \mathrm{~mA}$ rather than 4-20mA. The flow instrument may need recalibration, but the mis-calibration does not affect the non-linearity diagnosis. The valves were free of hard non-linearities such as stiction and did not become saturated. Figure 3 is a signal flow graph derived from mechanistic modeling showing which variables have causal interactions. Figure 3 also shows the distortion factors $D$ and the non-linearity statistics $N$, where $N>1$ means the measurement came from a non-linear time series and $D$ is larger if the time series has more harmonics (taken to be a sign of nonlinearity).

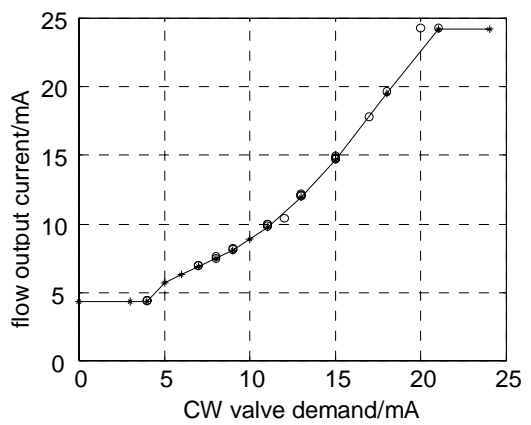

Figure 2. Non-linear characteristic of the cold water valve. The tests were centered at $12 \mathrm{~mA}$

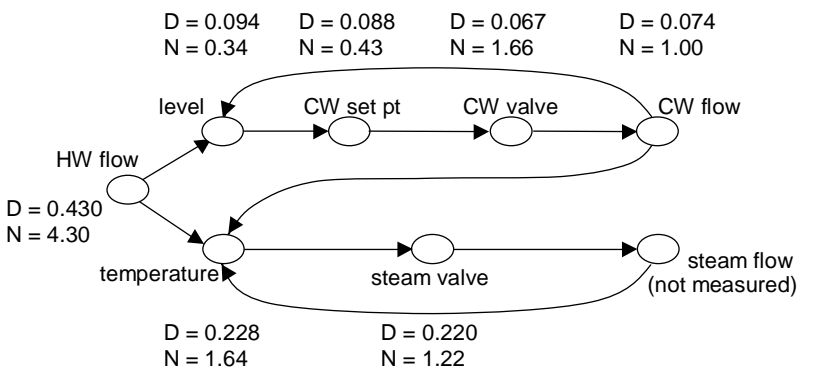

Figure 3. Signal flow graph, with $D$ and $N$ values

The following points can be observed:

- The known source of non-linearity (the demand to the hot water valve) had the largest values of $D$ and $N$. Therefore the on-off switching of the hot water valve was correctly diagnosed as the root cause;

- The non-linearity in the hot water valve demand propagated to the temperature measurement and to the steam valve. The non-linearity in the steam valve demand, which was further from the root cause, was less than that in the temperature measurement. The result shows that the non-linearity signatures grow stronger closer to the root cause;

- The non-linearity did not propagate to the level measurement. It is concluded that the slow first order lag dynamics of the volume in the vessel removed the non-linearity entirely;
- The $D$ and $N$ measures mostly agreed about the relative non-linearity of the time series;

- The cold water valve demand and cold water flow measurement were more non-linear than the level measurement and flow set point. Therefore the analysis identified a second source of non-linearity;

- $\quad$ The $D$ and $N$ measures disagreed about the relative non-linearity of the cold water valve and the cold water flow. Feedback is supposed to reduce nonlinearity compared to the open loop case. Therefore since the cold water flow is under feedback control the flow should be like the set point (i.e. linear) and the influence of the valve non-linearity should be taken up by the manipulated variable, i.e. the cold water valve demand. Such reasoning suggests the $N$ measure is correct because it shows the cold water flow was on the linear/non-linear threshold while the cold water valve demand was non-linear. Therefore the $N$ measure may be more responsive than the $D$ measure when mild non-linearity such as that in Figure 2 is involved.

\section{An industrial study}

A set of refinery data (courtesy of a SE Asian refinery) were examined. Figure 4 shows the three dimensional scores plot from spectral PCA analysis of the measurements. Three principal components explained $90 \%$ of the variability of the spectra and detected clusters. The white symbols represent distinct plant-wide disturbances. Figure 5 plots the time trends and spectra of the cluster represented by the white square symbols. All of them show a strong spectral peak at $0.06 \mathrm{~min}^{-1}$ corresponding to oscillations with a period of $16.7 \mathrm{~min}$. The results show that spectral PCA had the ability to detect a plant wide oscillatory disturbances. The locations of tags participating in the plant wide disturbance are shown in figure 6 as far as they are known by the authors. The black symbols are all close to the origin of the plot and represent tags dominated by noise having no distinctive spectral features.

The following points can be observed:

- The source of non-linearity is one of tags 13,33 or 34 because they had the largest $N$ and also large values of $D$. The information available on tag 13 is that it is a level control while 33 and 34 are flow instruments. Therefore attention should be focused on the flow valves that control tags 33 and 34 in the PSA unit. The basis for that recommendation is that faulty flow actuators are a common cause of control problems in the process industries (Ender, 1993; Desborough and Miller, 2001).

- The oscillation caused by the non-linearity propagated widely. It influenced many of the tags in the reformer and PSA units. The reason for its widespread influence is the off-gas recycle from the PSA unit to the reformer. 
- Analysis using spectral PCA showed that the disturbance reached as far back upstream as tag 25 . It would be tempting to think that tag 25 was the source of the disturbance because it is furthest upstream but non-linearity analysis showed clearly that Tag 25 was a propagated effect, not the root cause. It is not known how tag 25 was influenced by the disturbance. Possibilities are that tail gas flow is a manipulated variable in a control loop which regulates pressure in the process gas line, or that tail gas is recycled from another unit downstream from the PSA unit.

- Tags 3, 4, 19, 24 and 25 were disturbed by the oscillation but no non-linearity was detected. Therefore these tags are the furthest away from the root cause in terms of its propagated effects. For instance, Tag 19 is physically close to tag 34 but the disturbance would reach 19 via the off-gas recycle. Tag 25 requires more discussion because its distortion factor $D$ was not small, see comments below.

- The $D$ and $N$ measures both identified the same candidates for the root cause but disagreed about other loops, particularly on loop 25 which had $D=0.44$ but no evidence of non-linearity. An explanation is that the $D$ measure compares the power in the fundamental and the harmonics but does not take account of noise which is spread across all frequencies. By contrast, a test on a non-linear signal within random noise gives surrogates that are similarly random and the nonlinearity will therefore not be detected. This is what has happened with tag 25 , which is noisy. A pragmatic view is that a non-linear time series lost in noise is of no concern because if any maintenance action were required it would need to focus first on reducing the noise. Thus the conclusion is that the $N$ measure is better than $D$ because it gives a more useful result when noise is present.

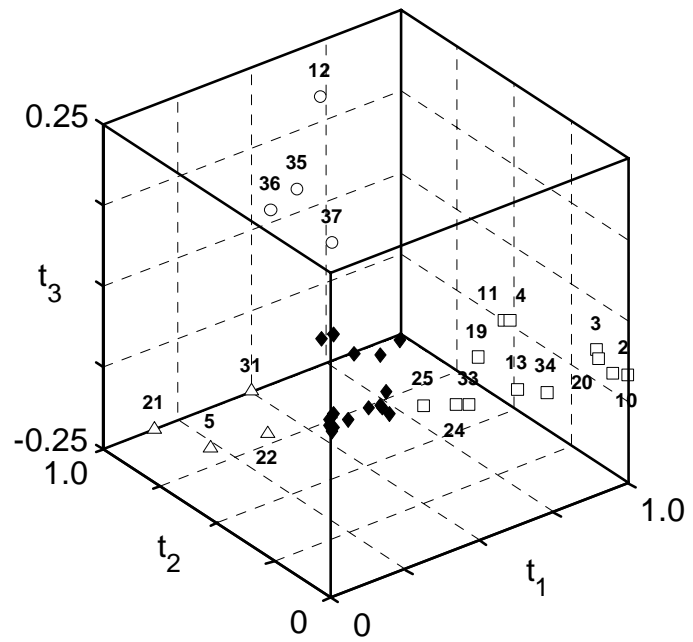

Figure 4. Spectral PCA scores plot. Each tag maps to a point in the three-dimensional space
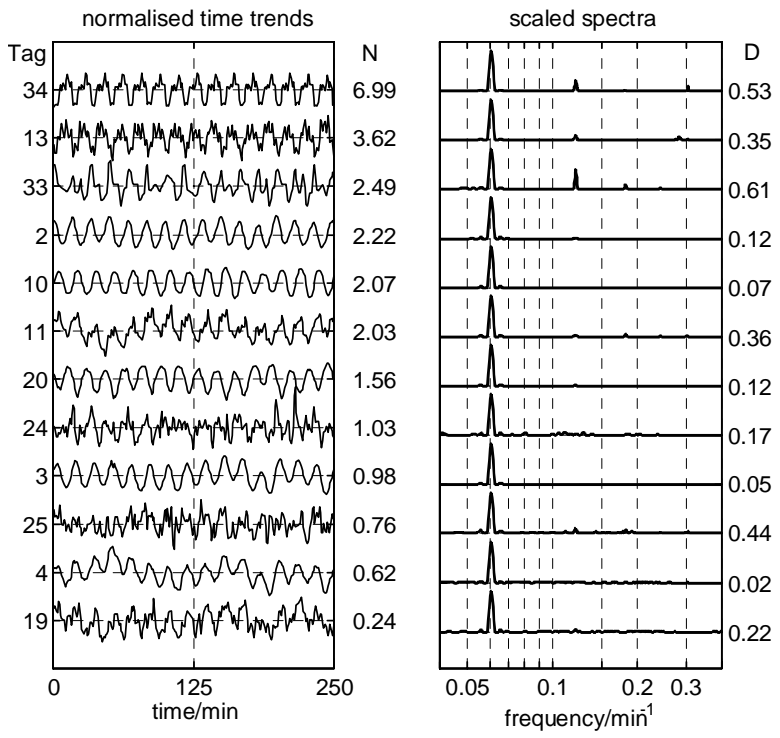

Figure 5. Time trends and spectra for the tags with square symbols with values of $N$ and $D$.

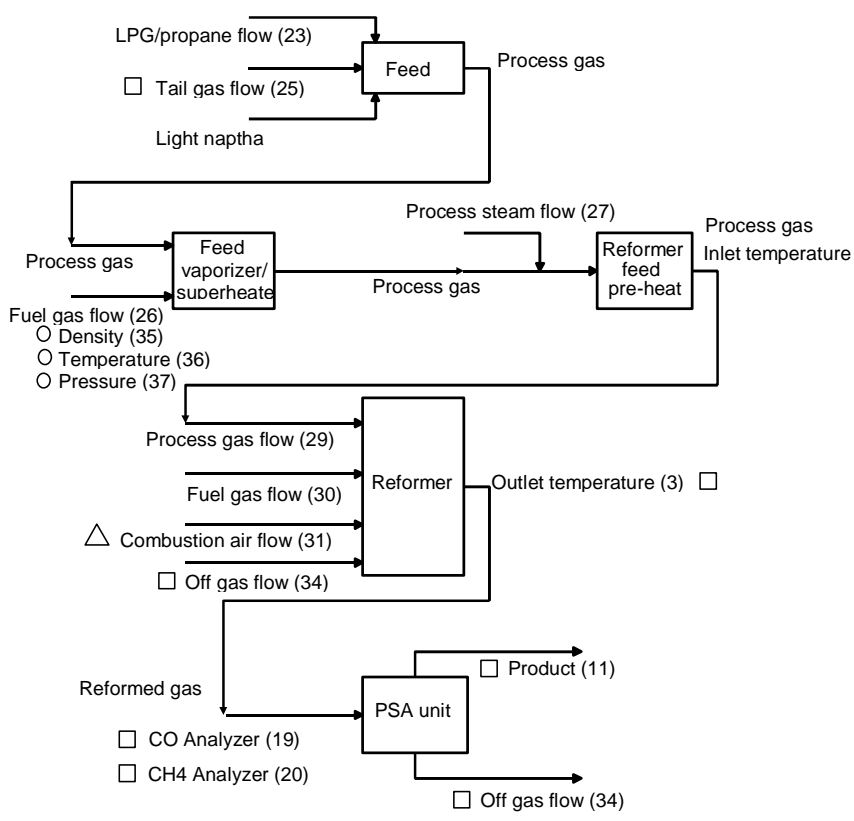

Figure 6. Process schematic for the industrial case study. The symbols relate to the clusters detected in figure 4. 


\section{Conclusion}

The paper has outlined an approach to the detection and diagnosis of plant-wide oscillations. Spectral PCA identified a group of measurements participating in a plantwide disturbance.

Two signatures for diagnosis of the source of an oscillatory disturbance were presented. Their performance was compared on experimental data where the root cause was known and in an industrial example where the root cause was inferred. Since non-linearity in control loops often leads to limit cycles with harmonics, the distortion factor $D$ was used to determine the harmonic content. The other method performed a non-linearity test using surrogate data giving a measure $N$. Both $D$ and $N$ were able to determine root causes and both were useful. The experimental results suggested $N$ was more sensitive to gentle non-linearity such as a curved valve characteristic. The industrial study showed $D$ could detect harmonic content in the presence of noise when $N$ did not detect the non-linearity. However, such a result might be misleading because it would be the noise and not the non-linearity which would limit the utility of that measurement for control or other purposes. Therefore the $N$ statistic would give a more appropriate result than the distortion factor $D$.

\section{Acknowledgements}

Nina Thornhill gratefully acknowledges the financial support of the Royal Academy of Engineering (Foresight Award) and support from members of the CPC group of the University of Alberta for this collaborative study. The authors would like to thank Shoukat Choudhury and R. Bushan Gopaluni for help with conducting the experimental runs for the study.

\section{References}

Åström, K.J. (1991). Assessment of achievable performance of simple feedback loops. Int. J. Adap. Control and Sig. Proc. 5, 3-19.

Cook, P.A. (1986). Nonlinear dynamical systems. Prentice-Hall International, London.

Chatfield, C. and A.J. Collins (1980). Introduction to multivariate analysis. Chapman and Hall, London, UK.

Desborough, L. and R. Miller (2001). Increasing customer value of industrial control performance monitoring -
Honeywell's experience. Preprints of CPC6, Tucson, Arizona.

Ender, D.B. (1993). Process control performance: Not as good as you think. Control Engineering (Sept), 180-190.

Goodwin, G.C. and J.S. Welsh (1999). Analysis of a novel method of autotuning a multivariable plant based on quantisation. Proc. ACC., San Diego, 3347-3351

Hägglund, T. (1995). A control-loop performance monitor. Control Eng. Practice, 3, 1543-1551.

Harris, T.J., C.T. Seppala, P.J. Jofreit and B.W. Surgenor (1996). Plant-wide feedback control performance assessment using an expert system framework. Control Engng. Prac., 9, 1297-1303.

Hegger R, H. Kantz and T. Schreiber (1999). Practical implementation of nonlinear time series methods: The TISEAN package. Chaos, 9, 413-435

Horch, A. (1999). A simple method for detection of stiction in control valves. Control. Engng. Prac., 7, 1221-1231.

Kantz, H. and T. Schreiber (1997). Nonlinear time series analysis. Cambridge University Press, Cambridge, UK.

Kaplan, D.T. (2000). Matlab software for nonlinear time series analysis. http://www.macalester.edu/ kaplan/software.

Ljung, L. (1999). System identificatioin for the user. Prentice Hall, New Jersey, USA.

Martin, G.D., L.E. Turpin L.E. and R.P. Cline (1991). Estimating control function benefits. Hydrocarbon Processing (June), 68-73.

McMillan, G.K. (1995). Improve control valve response. Chem. Eng. Prog. (June), 77-84.

Rengaswamy, R. and V. Venkatasubramanian (1995). A syntactic pattern-recognition approach for process monitoring and fault-diagnosis. Engng. Appl. Artificial Intell. 8, 35-51.

Qin, S.J. (1998). Control performance monitoring - a review and assessment. Comput. Chem. Eng., 23, 173-186.

Schreiber, T., and A. Schmitz (2000). Surrogate time series. Physica D., 142, 346-382.

Sharif, MA., and R.I. Grosvenor (1999). Sensor-based performance monitoring of a control valve unit. Proc. Inst. Mech. Eng. Part E-J. Process Mech. Eng. 213, 71-84.

Theiler, J., S. Eubank, A. Longtin, B. Galdrikian, B. and J.D. Farmer (1992). Testing for nonlinearity in time-series - the method of surrogate data. Physica D, 15, 77-94.

Thornhill, N.F., and T. Hägglund (1997). Detection and diagnosis of oscillation in control loops. Control Engng. Prac., 5, 1343-1354.

Thornhill, N.F., S.L. Shah and B. Huang (2000). Detection and diagnosis of unit-wide oscillations. PCI 2000, Glasgow, 26-28 July.

Valle, S., W.H. Li and S.J. Qin (1999). Selection of the number of principal components: The variance of the reconstruction error criterion with a comparison to other methods. Ind. Eng. Chem. Res, 38, 4389-4401. 